\title{
Polices et violences urbaines : la loi et le désordre dans les villes anglo-saxonnes
}

Jean-Claude Monet

\section{(2) OpenEdition \\ 1 Journals}

\section{Édition électronique}

URL : http://journals.openedition.org/conflits/638

DOI : $10.4000 /$ conflits. 638

ISSN : $1777-5345$

Éditeur :

CCLS - Centre d'études sur les conflits lilberté et sécurité, L'Harmattan

Édition imprimée

Date de publication : 16 juillet 1992

ISSN : 1157-996X

Référence électronique

Jean-Claude Monet, « Polices et violences urbaines : la loi et le désordre dans les villes anglosaxonnes », Cultures \& Conflits [En ligne], 06 | été 1992, mis en ligne le 06 janvier 2003, consulté le 30 mars 2021. URL : http://journals.openedition.org/conflits/638; DOI : https://doi.org/10.4000/conflits. 638

Ce document a été généré automatiquement le 30 mars 2021.

Creative Commons License 


\title{
Polices et violences urbaines : la loi et le désordre dans les villes anglo- saxonnes
}

\author{
Jean-Claude Monet
}

1 Les désordres survenus ces derniers mois dans certaines banlieues en France, une relative multiplication des situations de violences collectives entre policiers et jeunes en majorité issus des immigrations maghrébines, africaines ou antillaises, ont conduit certains observateurs à se demander si notre pays n'allait pas connaître des éruptions comparables à celles des villes américaines dans les années 1960, celles des inner-cities anglaises des années 1980 ou encore celle qui vient de ravager les quartiers populaires de Los Angeles. Avant de chercher à répondre de façon trop hâtive à la question de savoir si la France est menacée d'une vague d'émeutes urbaines, il est nécessaire de se doter d'instruments d'analyses à partir d'une comparaison entre les événements survenus de part et d'autre de l'Atlantique au cours des vingt-cinq dernières années.

2 Une observation rapide montre que les émeutes se produisent toujours dans des secteurs urbains où prévalent des rapports conflictuels entre la police et les minorités culturelles qui y résident. Les violences résultent la plupart du temps d'une action policière parfois brutale mais souvent banale et routinière. Les émeutes de Brixton en avril 1981, par exemple, partiront de l'intervention de deux agents en patrouille voulant transporter à l'hôpital un jeune Antillais blessé par d'autres jeunes Antillais. Les rumeurs, qui déforment ou amplifient la nature des interventions policières et leur signification, jouent un rôle essentiel dans le processus de mobilisation collective des résidents. Toujours à Brixton, la rumeur signalera que des policiers ont blessé un Antillais et qu'ils le conduisent au commissariat. Par la suite, la rumeur - non fondée annoncera le décès du blessé.

Avec l'entrée en scène de nouveaux acteurs, la protestation collective perd sa pureté originelle et la violence prend un aspect éclaté en même temps qu'elle se généralise. Certains manifestants continuent de vouloir se battre avec la police, d'autres cherchent à régler des comptes avec quelques commerçants du quartier, d'autres profitent de 
l'occasion pour acquérir des biens matériels qu'ils ne peuvent s'acheter, d'autres enfin, profitent de l'ambiance carnavalesque de ce temps et de cet espace hors normes. Les désordres durent deux ou trois jours, aggravés par l'improvisation, le manque d'équipement ou de professionnalisme des corps policiers, et leur propension à transformer le "maintien de l'ordre" en guerre personnelle. On parlera parfois d'"émeutes policières" tant les comportements policiers paraissent placés sous le signe de la vengeance plutôt que par la préoccupation de rétablir la paix publique ${ }^{1}$. Dans les mois qui suivent, sous le prétexte du "retour à l'ordre", les policiers multiplient les opérations de représailles, provoquant ainsi le surgissement de nouvelles émeutes.

4 Les réactions des autorités et de l'opinion oscillent entre une interprétation "conservatrice" et une interprétation "progressiste". La première met en cause l'irrationalité des masses en général et des "Noirs" en particulier ${ }^{2}$. La seconde met l'accent sur des facteurs macro-structurels tels que le chômage, la dégradation urbaine, le déclin ou l'insuffisance des budgets sociaux, le racisme ambiant. A aucun moment le rôle de la police n'est mis en cause, et les adversaires politiques se rejoignent toujours pour assurer les "forces de l'ordre" de leur confiance et de leur soutien³.

5 Certaines émeutes déboucheront sur l'institution de commissions d'enquête et la publication de rapports officiels qui dénoncent des pratiques policières abusives génératrices de ressentiment et d'hostilité chez les populations concernées. Mais le lien établi entre police et émeutes urbaines reste ténu : la police n'est ici que l'étincelle qui met le feu à un baril de poudre, et la principale cause des émeutes réside dans une accumulation de frustrations socio-économiques, dont il est "naturel" que parvenues à un certain point elles provoquent une explosion.

6 Des deux côtés de l'Atlantique les chercheurs ont consacré une énergie considérable pour essayer de produire des analyses rendant compte de ces formes d'action collective qui entrent malaisément dans le schéma des conflits sociaux ou politiques propres à l'ère industrielle. On sait que deux approches concurrentes se sont disputées le marché intellectuel: les théories de la frustration relative ou absolue - et celles de la mobilisation des ressources. Sans entrer dans le débat quant aux mérites et démérites respectifs de ces constructions ${ }^{4}$, on peut relever que l'une et l'autre ne consacrent qu'une place médiocre au rôle de la police dans le déclenchement des émeutes. Aucune des interprétations produites ne permet de répondre à des questions comme celles-ci : qu'est-ce qui entraîne le fonctionnement policier à déboucher sur des attitudes et des comportements qui provoquent l'hostilité générale des populations des ghettos et des inner-cities? D'où provient le changement de représentation des minorités qui les conduit à ne plus tolérer des comportements policiers moins extrêmes que dans le passé ? Comment s'effectue le passage de multiples frustrations individuelles à une action collective, alors même qu'il n'existe aucun "entrepreneur" susceptible de lancer un appel à la mobilisation? Quelle peut être la signification d'une violence collective qui ne vise aucune finalité instrumentale, ne peut déboucher sur aucune négociation, et dont les effets en fin de compte sont largement contre-productifs pour ceux qui l'initient?

$7 \quad$ Il faut partir de ce qui est le plus évident et de ce qui est exprimé avec le plus de force au sein de la population où se recrutent les émeutiers, aussi bien aux Etats-Unis qu'en Grande-Bretagne à savoir une hostilité extrême et largement répandue à l'égard de la police, des pratiques de ses agents et de ce qu'elle représente. Dans toutes les enquêtes conduites auprès des émeutiers, la dénonciation des pratiques policières passe toujours 
avant celle du chômage, de l'inconfort du logement, de la pauvreté des équipements sociaux. L'expression de cette hostilité s'organise autour de trois griefs : des contrôles incessants et discriminatoires de la part de la police; des comportements policiers violents et racistes lors des contrôles, des arrestations et des manifestations ; l'absence de protection offerte par la police aux victimes d'attaques raciales et face à la criminalité en général.

8 La présence massive de la police paraît à ce point boucher l'horizon des groupes sociaux concernés qu'il m'apparaît que ces violences collectives doivent être analysées avant tout comme des émeutes anti-policières. La police n'est pas seulement l'étincelle qui met le feu aux poudres, mais elle est au coeur du processus qui conduit de la frustration à la protestation et de la mobilisation à la violence collective. Pour dire les choses autrement : il me semble que les émeutes urbaines, résultent de la convergence de trois processus et comportent trois significations aux yeux des acteurs concernés ${ }^{5}$ : une réaction de protestation contre des stratégies policières discriminatoires, parce qu'elles reposent implicitement ou explicitement sur une association étroite entre "race et crime" une réaction d'indignation contre des pratiques policières abusives qui semblent moins rechercher l'application de la loi que l'infériorisation systématique d'un groupe ethnicisé ; une réaction d'affirmation d'une identité collective construite contre une société "blanche" environnante perçue comme injuste et dont la police constitue le symbole le plus visible.

La racialisation de la criminalité

9 L'association entre "race, ou "ethnicité" et criminalité est habituelle dans toutes les polices pour lesquelles le criminel vient toujours "d'ailleurs".

Historiquement elle a toujours été forte aux Etats-Unis et en Grande-Bretagne, pays où la culture policière entretient des affinités étroites avec les valeurs et la xénophobie des "parties respectables" des classes populaires. Cette xénophobie partagée constitue sans nul doute l'un des ressorts de l'identification symbolique entre la police et la "communauté", qui domine dans la tradition anglo-saxonne.

11 Mais les choses prennent un relief particulier lorsqu'au tournant des années 50 aux Etats-Unis et des années soixante en Grande-Bretagne, les statistiques de la police montrent une croissance rapide de la criminalité urbaine. Aux Etats-Unis, le nombre des vols, des cambriolages et des meurtres double entre 1960 et 1970. En GrandeBretagne, le total des délits enregistrés par la police passe de 750000 en 1960, à 2,5 millions en 1979, et 3,5 millions en 1985. Simultanément, les statistiques mettent en relief la baisse du nombre d'affaires délictueuses élucidées par la police.

Dans ces deux pays ${ }^{6}$, on a affaire à des corps policiers décentralisés, disposant d'une large autonomie opérationnelle, où les indicateurs quantitatifs d'activité jouent un rôle plus important qu'en France, par exemple, car ils permettent aux autorités locales d'exercer un contrôle indirect sur les responsables policiers, et à ceux-ci de légitimer leurs demandes d'allocations budgétaires. Etats-Unis et Grande-Bretagne constituent enfin deux pays où les chefs de police sont particulièrement sensibles à la façon dont la presse rend compte de leurs activités. I1 va donc se produire une forte pression à la fois interne et externe pour accroître la mobilisation policière et infléchir les tendances achetées par les courbes statistiques. A la même époque, ces polices se modernisent. Les bureaux de police périphériques jugés peu rentables sont supprimés et la centralisation opérationnelle renforcée. Les patrouilles à pied et la figure classique du "Bobby on the beat" disparaissent au profit de patrouilles motorisées, qu'on suppose plus efficaces car 
elles couvrent davantage de terrain. Ilotage et patrouilles pédestres se raréfient d'autant plus dans certains secteurs que les policiers s'y sentent mal à l'aise en raison des incidents qui s'y multiplient. En même temps que les relations avec la population se distendent, les flots habituels d'informations criminelles se tarissent, ce qui conduit les responsables policiers à développer des stratégies plus offensives dans la lutte contre la délinquance de rue.

Ces tactiques policières agressives touchent directement des populations durement marquées par le chômage, la disparition des emplois à faible qualification, l'insuffisance ou la régression des budgets sociaux. Aux Etats-Unis dans les années soixante, malgré le contexte général de prospérité économique, le taux de chômage frappe les "Noirs" deux fois plus que les "Blancs". Dans certains secteurs urbains, il n'est pas rare de trouver un taux de chômage de 33 \% au sein de la population active d'origine afro-américaine, soit 8,8 fois plus que le taux national moyen. En 1966, 11,9 \% des "Blancs" et 40,6 \% des "Noirs" vivent en dessous du seuil légal de pauvreté. En Grande-Bretagne, frappée par la désindustrialisation et la récession économique, le chômage s'accroît de 82 \% au sein de la population active issue de l'immigration entre 1979 et 1981. A Handsworth, au moment des émeutes, le taux de chômage atteint 50 \% chez les moins de 24 ans. On estime alors qu'un Anglais sur cinq trouve immédiatement un emploi en quittant l'école, mais ce pourcentage n'est que de un sur vingt chez les Antillais. Enfin, dans le cadre des compressions budgétaires mises en oeuvre par les Conservateurs, le pourcentage des dépenses sociales devient en Grande-Bretagne le plus faible des pays de la CEE.

14 Il se développe alors toute une économie souterraine à base de délits d'appropriation et de trafics divers - dont celui de la drogue qui sont généralement le fait de jeunes adultes et de mineurs. Cette économie parallèle procure des revenus indispensables qui permettent d'améliorer l'existence quotidienne. Les parents, bénéficiaires directs ou indirects du système, sont complices ou ferment les yeux ${ }^{7}$. Or, dans le cadre du durcissement général de ces stratégies, et parce que ce sont des infractions qui par nature donnent un taux de $100 \%$ en matière d'élucidation, la police

va se focaliser sur des infractions relativement mineures comme les ventes illicites de boisson, les fermetures tardives de boîtes de nuit, les petits trafics d'héroïne ou de cannabis $^{8}{ }^{9}$. Des comportements antérieurement tolérés vont être pourchassés du jour au lendemain. Dans la mesure où cette délinquance constitue une sorte de stratégie de survie, tout renforcement des pressions policières apparaît comme une menace quasiment vitale pour ceux qui s'y livrent. Les statistiques criminelles permettent aussi à la police de mettre en évidence le fait que la délinquance "de masse" provient, pour une large part, des secteurs urbains où vivent des concentrations importantes d'Afroaméricains ou d'Antillais, et que ces populations sont arrêtées, condamnées, emprisonnées de façon disproportionnée par rapport à leur volume dans la population globale. Au début des années 1970, les statistiques indiquent qu'aux Etats-Unis, environ 2 millions d'Afro-américains soit $8 \%$ de la population "noire" sont arrêtés chaque année par la police. Les données policières montrent en particulier que les jeunes "Blacks" se

16 retrouvent massivement impliqués dans le "mugging", c'est-à-dire le vol avec violence dans la rue. Il s'agit d'une forme de délit dont le volume global est relativement modeste mais qui suscite de fortes appréhensions dans l'opinion publique. La presse populaire sensationnaliste, reprenant les données communiquées par la police, va 
contribuer à faire du "black mugger" ou du "black criminal, rapist and burglar", la figure emblématique sur laquelle vont venir se cristalliser les angoisses que suscitent dans de larges secteurs de l'opinion les difficultés socio-politiques de l'heure. De leurs côtés, les corps policiers vont utiliser le thème du contrôle nécessaire de la "criminalité noire" pour redorer une image ternie par leur incapacité à contrôler la croissance de la délinquance, et obtenir des ressources supplémentaires. Dans le même temps, se renforce et se diffuse dans leurs rangs la conviction selon laquelle les "Noirs" sont asociaux, violents et qu'il convient de mettre en oeuvre contre eux des méthodes de contrôle spécifiques. Aux Etats-Unis, comme en Grande-Bretagne, il a toujours été d'usage d'expulser les minorités ethniques et les membres des classes dangereuses des quartiers où aux yeux des policiers ils ne sont pas à leur place. Cette vieille recette va naturellement être appliquée dans le cas d'une surveillance renforcée des jeunes "Noirs", systématiquement refoulés, vers leurs secteurs d'habitation ${ }^{10}$.

Ce processus de ghettoïsation a pour conséquence première d'accroître le volume de la délinquance dans des secteurs désignés, désormais, comme "risk areas". La géographie criminelle ainsi construite par la police justifie, dans un deuxième temps, la mise en oeuvre de stratégies agressives appuyées sur des unités spécialisées : les Special Patrol Groups en Grande-Bretagne et les Aggressive Patrol Groups aux Etats-Unis. Désormais, certains quartiers paraissent mis en état de siège périodique par des escouades motorisées et armées, qui opèrent des débarquements en force dans les secteurs réputés criminogènes. Une opération de saturation du terrain baptisée "Swamp 81" se déroulera à Brixton du 6 au 11 avril 1981; $50 \%$ des personnes contrôlées par la police seront des Antillais, alors qu'ils ne représentent que $12 \%$ de la population du secteur, et $2 / 3$ des contrôlés auront moins de 21 ans ${ }^{11}$. Cette opération policière sera directement à l'origine des émeutes qui surgiront dans ce secteur londonien ${ }^{12}$.

Du point de vue des policiers, l'intensification des contrôles de jeunes antillais ou afroaméricains apparait comme une stratégie statistiquement rationnelle, puisque ceux-ci sont plus fréquemment impliqués dans certaines formes de criminalité de rue redoutées par l'opinion publique que d'autres groupes sociaux. Mais il existe un fossé que la police n'hésite pas à franchir - entre l'affirmation que les "Noirs" sont proportionnellement plus impliqués que les "Blancs" dans des affaires criminelles, et le processus qui transforme chaque "Noir" en suspect ${ }^{13}$. Cette tendance à la suspicion généralisée sur la base de critères biologiques, et non pas de l'exercice du "flair" policier, s'explique en partie par le fait qu'aux Etats-Unis - surtout à cette époque - et en Grande-Bretagne, les corps policiers sont composés majoritairement de "Blancs"14. Ne vivant pas dans les mêmes quartiers que les communautés "noires", ils sont incapables de faire la différence entre les citoyens respectueux des lois et les délinquants - en particulier lorsqu'il s'agit de jeunes - distinction qu'ils sont habituellement en mesure d'opérer lorsqu'ils interviennent dans des quartiers habités majoritairement par des populations blanches"15.

19 Parce qu'elles se déroulent sur une vaste échelle, ces opérations alimentent de façon substantielle les statistiques d'arrestation. A Londres, les chercheurs considèrent qu'elles représentent environ $1 / 4$ de toutes les arrestations opérées par la police. Mais, opérés sur la base de critères incertains, ces contrôles n'ont qu'un taux de productivité faible : seuls 3 à $4 \%$ d'entre eux débouchent sur des arrestations ${ }^{16}$. Autrement dit, 96 à $97 \%$ de personnes n'ayant en principe rien à se reprocher, auront subi des tracasseries policières de nature à les irriter. Du côté des groupes qui les subissent, ces stratégies 
paraissent d'autant plus injustes et discriminatoires que les policiers, à l'évidence, n'opèrent pas de la même façon dans les quartiers "blancs" et qu'ils n'utilisent pas les mêmes techniques. A Los Angeles, des analyses statistiques récentes ont montré, par exemple, qu'il existait un fort degré de corrélation entre l'utilisation de patrouilles avec des chiens policiers et la composition majoritairement non "blanche" de certains secteurs.

La principale source de ressentiment pour les minorités afro-américaines et antillaises ne résulte pas tant d'une présence policière pesante dans leurs territoires, ni même de la multiplication des contrôles qu'ils subissent, mais du fait que ceux-ci leur sont appliqués de façon systématique et selon des techniques discriminatoires, puisque la police se garde bien d'utiliser les mêmes procédés dans les quartiers résidentiels habités par des "Blancs". En définitive, ces stratégies policières témoignent à leurs yeux du passage d'une discrimination statistique à une discrimination raciale et elles mettent en évidence le caractère raciste de l'institution policière dans son ensemble.

La reproduction du modèle colonial

21 La logique institutionnelle, que je viens de décrire, fonctionne à un niveau trop global pour rendre compte à elle seule de la force des représentations négatives des jeunes afro-américains et des jeunes antillais à l'égard de la police. Les témoignages recueillis au cours des enquêtes montrent, en effet, que ces perceptions ne relèvent ni de l'ordre du fantasme, ni d'un effet de halo, mais qu'elles s'enracinent dans des expériences vécues. De façon récurrente, les situations de rencontre avec les policiers sont décrites par les jeunes "Blacks" comme placées sous le signe d'une arrogance, d'une brutalité et d'une violence que l'appartenance ethnique de ceux qui en sont les victimes suffirait à expliquer ${ }^{17}$.

Les études américaines et anglaises convergent pour indiquer qu'effectivement, les préjugés, les discours racistes et les attitudes hostiles aux "Noirs" sont choses courantes chez les policiers ${ }^{18}$. Une enquête conduite au milieu des années 1960 auprès des polices de Boston, Chicago et Washington, indique que $75 \%$ des policiers sont à classer comme "très" ou "considérablement" racistes ${ }^{19}$. A Manchester, dans le quartier de Moss Side, les enquêteurs ont également montré que des expressions comme "nègres", "bâtards", constituaient des modes usuels d'interpellation de jeunes Antillais. Quant à la brutalité de la police américaine, et sa propension à utiliser des armes à feu contre les Afro-américains, elle est largement documentée. Une étude conduite dans 7 villes américaines au milieu des années 1970 a montré que $79 \%$ des citoyens tués par des policiers étaient des Afro-américains alors qu'ils ne constituaient que $39 \%$ de la population de ces villes.

Le comportement des policiers, dans les rencontres de rue, est fonction de la façon dont ils définissent la situation et anticipent son évolution. Or définition et anticipation sont largement fonction des catégories dans lesquelles les policiers font entrer les citoyens qui sont en face d'eux ${ }^{20}$. Dans son étude pionnière d'un grand corps de police américain dans les années cinquante, Westley indique que, pour les policiers, il existe trois grandes catégories suspectes, les habitants des slums, les "Noirs" et les criminels de profession. Tous constituent des populations dépourvues de moralité, ignorant la loi et n'ayant aucun respect pour la police. La brutalité avec eux est possible, car ce sont des gens sans influence politique. Elle est nécessaire, car ils sont tous potentiellement coupables, et par la violence la police peut leur arracher des aveux sur des méfaits qu'elle ignore. Elle est justifiée, car les policiers se considèrent comme supérieurs à ces 
gens-là, et pensent que s'ils n'imposent pas leur supériorité par la force, leur autorité ne sera pas reconnue. Dans la tradition policière britannique, le recours à la force s'opère sur une échelle beaucoup plus limitée et contrôlée. Mais les modes de catégorisation sont identiques et dans les deux pays, il est patent qu'avec les "Noirs" et surtout les jeunes, le policier s'attend généralement à des difficultés et à ce qu'ils manquent de ce "respect" auquel il attache le plus grand prix. De ce fait, il procédera plus facilement à des arrestations et utilisera plus fréquemment la force avec des catégories de population sans prestige et sans influence, considérées comme naturellement inférieures, sauvages, ou criminelles-nées.

Les policiers se défendent généralement contre les accusations de brutalité et de racisme, en faisant valoir que ces comportements abusifs ne sont le fait que d'une minorité d'entre eux. Mais cette argumentation est insuffisante pour deux raisons. La première tient à l'effet de boule de neige produit par un petit nombre de policiers discourtois ou racistes.

\section{NOTES}

1. Stark R., Police Riots. Collective violence and law enforcement, Belmont (California), Wadsworth Publishing Company 1972. Hawkins H. \& Thomas R., White policing of black populations. A history of race and social control in America., in Cashmore E. \& McLaughlin (eds) : Out of order ? Policing Black People, London, Routledge, 1991, pp. 64-86.

2. Aussi bien le rapport Kerner dans le cas de Detroit que le travail d'Oberschall sur les émeutes de Watts ont montré que ces violences collectives ne choisissent pas leurs cibles au hasard et qu'elles s'en prennent bien plus aux biens matériels qu'aux personnes.

3. Solomos J. \& Rackett T., Policing and urban unrest. Problem Constitution and policy response, in Cashmore E. \& McLaughlin (eds) : Out of order ? Policing Black People, London, Routletge, 1991, pp. 42-64.

4. Rude James B., Theories of civil violence. Berkeley and Los Angeles, University of California Press., 1988 ; également : Lapeyronnie D.,

\section{Mouvements sociaux et action politique.}

Existe-t-il une théorie de la mobilisation des ressources à Revue française de Sociologie, XXIX, 1988, pp. 593-619. 5. Reiner R., The politics of the police, Brighton, Wheatsheaf, 1985.

5. Pour une approche voisine de la mienne mais limitée au cas britannique, voir Jefferson T., Race, crime and policing. Empirical, theoretical and methodological issues. International Journal of the Sociology of Law (16),1988, pp. 521-529.

6. McKenzie I.K. \& Gallagher G. P., Behind the uniform Policing in Britain and America. Hemel Hanpsted \& New York, Harvester Weatsheaf \& St Martin's Press., 1989.

7. D'où la fréquente mise en cause par les sphères officielles d'un "laxisme parental", sans doute réel, mais décrit à tort comme un trait culturel spécifique. 
8. Des interventions policières de ce type vont constituer l'"incident déclencheur" de nombreuses émeutes, en particulier à Detroit et à Newark en 1967 à Bristol en 1980, à Birmingham en 1981.

9. Gilroy P., There ain't no black in the Union Jack, London, Unwin Hyman, 1987.

10. Cashmore E. \& McLaughlin F., Out of order ? in Cashmore E. \& McLaughlin E. (eds), Out of Order Policing black People, London, Routledge, 1991, pp. 10-41. Dunne J. G., Law and Disorder in Los Angelcs. The New York Review of Books, 10 octobre et 24 octobre 1991 (2parties), Part two, p. 58.

11. A ne s'en tenir qu'aux chiffres officiels, car dans la pratique - et en contradiction avec les instructions officielles - une masse importante de contrôles ne fait pas l'objet d'enregistrements (Smith \& Gray 1985).

12. Benyon J. (eds), Scarman and after Essays reflecting on Lord Scarman's report, the riots and their aftermath, Oxford, Pergamon Press, 1984. Jacobs B. D., The Brixton riots : London, 1981, in U. Rosenthal, M.T. Charlcs \& PT Hart (eds), Coping with crises, Thc managment of disasters, riots and terrorism, Charles C. Thomas, Publ. Springfield III 1989.

13. Smith D. J. \& Gray J., Polire and People in London : a survey of Londoners, London, Gower Publisher 1985.

14. Cashmore E., black Cops Inc., in Cashmore E. \& Mclaughlin (eds) : Out of Order? Policing Blach People, London, Routledge, 1991, pp. 87-108.

15. Oberschall A., Sorial conflict and social movements. Ch. 6 : Mobilization, leaders and followers in The Civil Rights movement in the United States, 1950-1970, Englewood Cliffs, N. J. Prentice Hall Inc., 1973.

16. Bien que peu productif le contrôle systématique et aléatoire des jeunes et des "Noirs" constitue une activité valorisée par la culture professionnelle des policiers parce qu'en assonance avec leur goût de l'action, de l'excitation, de la "chasse", de l'exercice de l'autorité. En bref il s'agit d'un "vrai travail policier". Sur la culture policière, voir l'ouvrage de Robert Reiner déjà cité.

17. Voir les rapports Kerner et Scarman.

18. Pour une revue critique de cette littérature, voir Monet J.C., Police et racisme, Communication au colloque international "Trois jours sur le racisme" (Créteil, 5-6-7juin 1991).

19. Flowers R.B., Minorities and Criminality Coll. Contributions in criminology and penology, Westport, Greenwood Press, 1988.

20. Westley WA., Violence and the police. A sociological study of law, custom, and morality Cambridge (Mass.) The MIT Press, 1970. Cain M., Society and the police man's role. London, Routledge, 1973.

\section{RÉSUMÉS}

Des émeutes urbaines comparables à celles survenues aux États-Unis dans les années 1960 - ou récemment à Los Angeles et en Grande-Bretagne dans les années 1980 sont-elles possibles en France ? Chercher à répondre à cette question suppose de repérer les logiques à l'oeuvre et les 
processus conduisant de l'accumulation de frustrations individuelles à une mobilisation et à des violences collectives. Un examen attentif montre que la police est au coeur de ces processus ; par les pratiques qu'elle met en oeuvre dans la répression de la criminalité ; en raison des attitudes racistes et des brutalités de certains de ses agents ; en raison, enfin, de son importance symbolique dans les sociétés modernes : elle est ce grâce à quoi et contre quoi se construisent et se développent les violences collectives. Les émeutes urbaines sont donc d'abord des émeutes antipolicières.

Could urban riots on the scope of those that swept through the United States in the sixties - and more recently in Los Angeles - or through Great Britain in the eighties, occur in France ? To answer this question, a study is made of the process of growth of individual grievances and their combination into a type of mobilisation producing mass violence. A careful examination shows that it is the police that stands in the centre of the process : through its methods of dealing with crime ; the racist attitude and brutality of some of its officers ; its symbolic role in modern societies : collective violence is created by and against it. Urban riots are in fact mainly antipolice riots.

\section{INDEX}

Mots-clés : droit, police, répression, villes/ sociologie urbaine, violence, loi

Index géographique : Etats-Unis, Royaume Uni

\section{AUTEUR}

\section{JEAN-CLAUDE MONET}

Policier à l'origine, est aujourd'hui chercheur au Centre d'Analyse et d'intervention sociologiques 